\title{
The influences on human longevity by HUMTHO1.STR polymorphism (Tyrosine Hydroxylase gene) A relative risk approach
}

\author{
Qihua Tan ${ }^{\mathrm{a}}$, D. Bellizzi ${ }^{\mathrm{b}}$, G. Rose ${ }^{\mathrm{b}}, \mathrm{S}$. Garasto ${ }^{\mathrm{b}}$, C. Franceschi ${ }^{\mathrm{c}}$, T. Kruse $^{\mathrm{a}}$, \\ J.W. Vaupel ${ }^{\mathrm{d}}$, G. De Benedictis ${ }^{\mathrm{b}}$, A.I. Yashin ${ }^{\mathrm{d}, \mathrm{e}, *}$ \\ a Department of Clinical Biochemistry and Genetics, KKA, Odense University Hospital, Odense, Denmark \\ ${ }^{\mathrm{b}}$ Cell Biology Department, University of Calabria, Rende, Italy \\ ${ }^{\mathrm{c}}$ Department of Experimental Pathology, University of Bologna, Bologna, Italy \\ d Max-Planck Institute for Demographic Research, Doberaner Str. 114, 18057 Rostock, Germany \\ ${ }^{\mathrm{e}}$ Center for Demographic Studies and Sanford Institute, Duke University, Durham, NC, USA
}

Received 27 February 2002; received in revised form 1 July 2002; accepted 17 July 2002

\begin{abstract}
A new method based on the recently developed relative risk approach is introduced, and applied to data from Italian centenarian study (965 subjects aged from 13 to 109 years old) for investigating influences on longevity by Tyrosine Hydroxylase $(\mathrm{TH})$ gene variability. The strategic parameterization enables the model to disentangle the various ways by which HUMTHO1.STR alleles (alleles 6, 7, 8, 9, 10*, 10, as defined according to the number of repeats) may contribute in reducing or increasing the hazard of death with different patterns of influences. Among all the alleles, we have found that allele $10^{*}$ (10 imperfect repeats) shows a remarkable dominant and beneficial effect that reduces the log hazard of death in an additive manner. The results confirm that HUMTHO1.STR polymorphism is involved in the modulation of human longevity. (C) 2002 Elsevier Science Ireland Ltd. All rights reserved.
\end{abstract}

Keywords: TH gene; Relative risk; Polymorphism; Longevity

\section{Introduction}

Life span is a complex trait, which is shaped by genetic, environmental, and stochastic factors. The genetic component has been intensively investigated by correlation studies in twins (McGue et

\footnotetext{
* Corresponding author. Tel.: +49-381-2081106; fax: +49381-2081169

E-mail address: yashin@demogr.mpg.de (A.I. Yashin).
}

al., 1993; Herskind et al., 1996; Yashin et al., 1999a), association studies in unrelated individuals (De Benedictis et al., 2001; Tan et al., 2001a, 2002a,b), and sib-pairs approach (Puca et al., 2001).

Increasing evidence shows that, as in model organisms, the capability to cope with stress in humans is also a leading factor in rate and quality of aging (Franceschi et al., 2000). Therefore, all the genes involved in the complex mechanisms which 
control stress response are longevity candidate genes. The Tyrosine Hydroxylase (TH) gene (11p15.5) encodes $\mathrm{TH}$, the rate-limiting enzyme in the synthesis of catecholamines, amino acidderived molecules produced by the sympathetic nervous system in response to stress. An STR marker (HUMTHO1.STR) lies inside TH intron 1 (Polymeropoulos et al., 1991) a region which is involved in RNA alternative splicing of the $\mathrm{TH}$ primary transcript for generating four tissuespecific types of mRNA (Kobayashi et al., 1988). Association between HUMTHO1 variability and longevity was firstly observed (De Benedictis et al., 1998). Thereafter, gene-environment interactions and sex-specific genetic effects have been observed when HUMTHO1 variability was analyzed by models that combine genotypic and demographic information in the estimation of genetic influence on life span (Tan et al., 2001b). The involvement of the $11 \mathrm{p} 15.5$ chromosomal region in the longevity phenotype has been confirmed by linkage disequilibrium studies carried out by analyses of two other polymorphic genes occurring in the same chromosomal region: Insulin and Insulin Growth Factor 2 (De Luca et al., 2001). However, the effects of HUMTHO1.STR allele-allele interactions on hazard of death and longevity have not been analyzed until now. The aim of the present study is to introduce an analytical method that permits to disentangle the effects of HUMTHO1.STR alleles on the longevity trait. The results confirm that this polymorphism is involved in the modulation of human longevity and that allele-allele interaction plays an important role.

\section{Materials and methods}

\subsection{Samples}

The data used in this study is an updated version of the data by which TH gene/longevity association was first discovered (De Benedictis et al., 1998). All individuals in the sample are unrelated, clinically healthy and are from various socioeconomic backgrounds. For each individual, origin and residence in Italy were checked by inter- view as far as the grandparents' generations. All individuals gave informed consent prior to their inclusion in the study. A total of 965 subjects were collected in the new update. Among them, 210 are centenarians who were over 100 years old when blood sample was taken. In the centenarians, 68 are males and 142 are females with a female to male sex ratio about 2.1. The remaining 755 subjects cover a wide range of ages (from 13 to 99 years) with a median age of 59 .

\subsection{DNA analysis}

DNA was extracted from blood buffy coats following standard procedures. HUMTHO1 typing was carried out on PCR amplified DNA fragments (De Benedictis et al., 1998) by GENESCAN software (ABIPRISM 310 Genetic Analyzer, Applied Biosystem) and designated according to the repeat number.

\subsection{Population survival data}

The method for data analysis is based on the survival distribution function for the population from which the sample has been taken. Since subjects in the sample were born into different birth cohorts, we used in the calculation birth cohort life tables corresponding to subjects' ages at participation. The Italian population survival data were provided by the Max-Planck Institute for Demographic Research in Rostock, Germany.

\subsection{Statistical method}

The statistical method we are going to apply is based on a relative risk approach that has been recently proposed and described by Yashin et al. (1998, 1999b, 2000), Tan et al. (2001b). We define the relative risk of one observed genotype $r$ as the ratio of hazard of death for carriers, $\mu(x, r)$, to that for the non-carriers or the baseline hazard, $\mu_{0}(x)$. Then, according to the proportional hazard assumption, $\mu(x, r)=r \mu_{0}(x)$. The corresponding survival function for the carriers is, 


$$
\begin{aligned}
S(x, r) & =\mathrm{e}^{-\int_{0}^{x} \mu(t, r) \mathrm{d} t}=\mathrm{e}^{-\int_{0}^{x} r \mu_{0}(t) \mathrm{d} t}=\mathrm{e}^{-r \int_{0}^{x} \mu_{0}(t) \mathrm{d} t} \\
& =\mathrm{e}^{-r H_{0}(x)}=s_{0}(x)^{r} .
\end{aligned}
$$

Here, $s_{0}(x)$ is the baseline survival distribution corresponding to the baseline hazard function, $H_{0}(x)$ is the cumulative hazard at age $x$. Although $r$ can take any value greater than zero, a genotype with $r$ larger than 1 increases the hazard of death, while a genotype with $r$ smaller than 1 reduces it.

For a given single allele, there are three genotypes that can be observed among the subjects, that is, individuals with no such allele designated as $(--)$, individuals with one such allele $(-+/+$ $-)$ and individuals with two such alleles $(++)$. If frequency of the allele is $p$, then the corresponding genotype frequencies can be calculated as $(1-p)^{2}$, $2 p(1-p)$ and $p^{2}$ according to the binomial distribution. Risks on hazard of death are defined for each genotype but on a relative base, that is, we take the genotype $(--)$ as the reference with relative risk 1, and relative risks for heterozygous $(-+/+-)$ and homozygous $(++)$ genotypes $r_{1}$ and $r_{2}$, respectively. Further, we define the risk of gene-sex interaction, $r_{\mathrm{g} \times \mathrm{s}}$, as the risk of male carriers to that of female carriers. Then the average survival at age $x$ for a mixed population is (Vaupel and Yashin, 1985)

$$
\begin{aligned}
\bar{s}_{\mathrm{m}}(x)= & p^{2} s_{0, \mathrm{~m}}(x)^{r_{2} r_{\mathrm{g} \times \mathrm{s}}}+2 p(1-p) s_{0, \mathrm{~m}}(x)^{r_{1} r_{\mathrm{g} \times \mathrm{s}}} \\
& +(1-p)^{2} s_{0, \mathrm{~m}}(x), \\
\bar{s}_{\mathrm{f}}(x)= & p^{2} s_{0, \mathrm{f}}(x)^{r_{2}}+2 p(1-p) s_{0, \mathrm{f}}(x)^{r_{1}} \\
& +(1-p)^{2} s_{0, \mathrm{f}}(x) .
\end{aligned}
$$

Here, $\bar{s}_{\mathrm{m}}(x)$ and $\bar{s}_{\mathrm{f}}(x)$ are survival rates at age $x$ for males and females obtainable from population statistics. The corresponding genotype frequencies at age $x$ for the three genotypes in males are ${ }_{\mathrm{m}} p_{2}(x)=\left(p^{2} s_{0, \mathrm{~m}}(x)^{r_{2} r_{\mathrm{g} \times \mathrm{s}}} / \bar{s}_{\mathrm{m}}(x)\right)$, $\left(2 p(1-p) s_{0, \mathrm{~m}}(x)^{r_{1} r_{\mathrm{g} \times \mathrm{s}}} / \bar{s}_{\mathrm{m}}(x)\right)$, ${ }_{\mathrm{m}} p_{1}(x)=$ $\left.p)^{2} s_{0, \mathrm{~m}}(x) / \bar{s}_{\mathrm{m}}(x)\right)$. In the same way, we can calculate ${ }_{\mathrm{f}} p_{2}(x),{ }_{\mathrm{f}} p_{1}(x)$ and ${ }_{\mathrm{f}} p_{0}(x)$ for females. Based on multinomial distribution (Evans et al., 1993), the likelihood function can be written as,

$$
L \propto \prod_{x} \prod_{i} p_{i}(x)^{\mathrm{m} n_{i}(x)}{ }_{\mathrm{f}} p_{i}(x)^{\mathrm{f}_{i}(x)} \quad i=0,1,2,
$$

where ${ }_{\mathrm{m}} n_{i}(x),{ }_{\mathrm{f}} n_{i}(x)$ are numbers of males and females at age $x$ in each of the three genotypes. Parameters that maximize the likelihood function are estimated by the maximum likelihood procedure in Gauss software (Aptech System, 1996).

There are two important features in this approach. First, no parametric form of the baseline survival function is imposed. The estimation procedure is based on a two-step algorithm described by Tan et al. (2001b). In this procedure, the sex-specific baseline survivals are calculated non-parametrically from Eq. (2) for given risk and frequency parameters. The so obtained distribution-free baseline survivals are then introduced in Eq. (3) for parameter estimation by maximizing the likelihood function. The procedure is iterated until the maximum likelihood is achieved. Second, the risk parameters for heterozygous and homozygous genotypes are specified separately, which allows for detecting recessive and additive effects in gene action.

\section{Results}

Table 1 shows the distribution of subjects according to age group, sex and HUMTHO1.STR genotype. Table 2 shows, for each allele, the estimated initial allele frequency and the relative risks by genotype. Significance levels for risk parameters (relative risks for the allele and for gene-sex interaction) were determined by testing the statistical differences between the estimated risks and 1 , with the null hypothesis $H_{0}: r=1$. The probability of a Type I error is $\alpha=0.05$.

Allele $10^{*}$ is interesting in that both heterozygous and homozygous genotypes display beneficial influences that reduce the carriers' hazards of death. Corresponding relative risks are 0.920 $\left(p_{- \text {value }}=0.013\right)$ and $0.851\left(p_{- \text {value }}=0.005\right)$ for the heterozygous and homozygous genotypes, respectively. However, after correction for multiple comparisons, we obtain a probability of making type I error $\alpha^{\prime}=1-(1-0.013)^{6} \approx 0.076$ for the risk of allele $10^{*}$ heterozygous genotype, that is borderline significant, while the risk for the $10 * 10 *$ homozygous genotype remains significant $\left(\alpha^{\prime}=0.030\right)$. Interestingly, the relative risk of the 
Table 1

Distribution of subjects by age group, sex and genotype

\begin{tabular}{|c|c|c|c|c|c|c|c|c|c|}
\hline \multirow[t]{2}{*}{ Genotype } & \multicolumn{2}{|c|}{$13-34$} & \multicolumn{2}{|c|}{$35-64$} & \multicolumn{2}{|c|}{$65-99$} & \multicolumn{2}{|l|}{$100+$} & \multirow[t]{2}{*}{ Total } \\
\hline & Male & Female & Male & Female & Male & Female & Male & Female & \\
\hline \multicolumn{10}{|l|}{ Allele 6} \\
\hline$(--)$ & 37 & 60 & 70 & 57 & 55 & 95 & 32 & 82 & 488 \\
\hline$(-+,+-)$ & 31 & 38 & 60 & 51 & 61 & 81 & 31 & 48 & 401 \\
\hline$(++)$ & 3 & 9 & 9 & 6 & 20 & 12 & 5 & 12 & 76 \\
\hline \multicolumn{10}{|l|}{ Allele 7} \\
\hline$(--)$ & 44 & 70 & 102 & 69 & 110 & 130 & 44 & 105 & 674 \\
\hline$(-+,+-)$ & 27 & 33 & 36 & 43 & 25 & 53 & 21 & 33 & 271 \\
\hline$(++)$ & 0 & 4 & 1 & 2 & 1 & 5 & 3 & 4 & 20 \\
\hline \multicolumn{10}{|l|}{ Allele 8} \\
\hline$(--)$ & 53 & 86 & 108 & 91 & 102 & 146 & 48 & 115 & 749 \\
\hline$(-+,+-)$ & 17 & 18 & 30 & 20 & 34 & 41 & 18 & 27 & 205 \\
\hline$(++)$ & 1 & 3 & 1 & 3 & 0 & 1 & 2 & 0 & 11 \\
\hline \multicolumn{10}{|l|}{ Allele 9} \\
\hline$(--)$ & 42 & 59 & 83 & 79 & 89 & 124 & 48 & 90 & 614 \\
\hline$(-+,+-)$ & 27 & 43 & 47 & 30 & 43 & 60 & 20 & 51 & 321 \\
\hline$(++)$ & 2 & 5 & 9 & 5 & 4 & 4 & 0 & 1 & 30 \\
\hline \multicolumn{10}{|l|}{ Allele $10^{*}$} \\
\hline$(--)$ & 49 & 76 & 83 & 69 & 88 & 111 & 45 & 73 & 594 \\
\hline$(-+,+-)$ & 21 & 27 & 53 & 41 & 43 & 65 & 20 & 56 & 326 \\
\hline$(++)$ & 1 & 4 & 3 & 4 & 5 & 12 & 3 & 13 & 45 \\
\hline \multicolumn{10}{|l|}{ Allele 10} \\
\hline$(--)$ & 66 & 102 & 135 & 111 & 134 & 184 & 68 & 134 & 934 \\
\hline$(-+,+-)$ & 5 & 5 & 4 & 3 & 2 & 4 & 0 & 7 & 30 \\
\hline$(++)$ & 0 & 0 & 0 & 0 & 0 & 0 & 0 & 1 & 1 \\
\hline
\end{tabular}

heterozygous genotypes for allele $10^{*}$ is approximately the square root of the risk of the $10 * 10^{*}$ homozygous genotype, thus suggesting a doseeffect for this allele. According to the model specification, allele $10^{*}$ phenotypic value in term of log hazard of death is additive on the estimated relative risk. In Fig. 1, we plot the observed and the estimated frequencies of alleles 8 and $10^{*}$ carriers by age. While frequency for allele eight carriers is almost constant (Fig. 1A), the frequency

Table 2

Estimated allele frequency and relative risks by genotype for each allele

\begin{tabular}{|c|c|c|c|c|c|c|c|c|c|c|}
\hline \multirow[t]{2}{*}{ Allele } & \multirow[t]{2}{*}{ Frequency } & \multicolumn{3}{|c|}{ Heterozygous genotype } & \multicolumn{3}{|c|}{ Homozygous genotype } & \multicolumn{3}{|c|}{ Gene-sex interaction } \\
\hline & & Risk & $\mathrm{SE}$ & $P$-value & Risk & SE & $P$-value & Risk & SE & $P$-value \\
\hline 6 & 0.290 & 1.055 & 0.034 & 0.107 & 1.058 & 0.055 & 0.295 & 0.906 & 0.042 & 0.025 \\
\hline 7 & 0.161 & 1.026 & 0.038 & 0.501 & 0.993 & 0.074 & 0.927 & 0.956 & 0.049 & 0.365 \\
\hline 8 & 0.118 & 1.039 & 0.043 & 0.356 & 1.147 & 0.141 & 0.298 & 0.899 & 0.049 & 0.040 \\
\hline 9 & 0.210 & 0.983 & 0.034 & 0.618 & 1.409 & 0.178 & 0.022 & 1.064 & 0.052 & 0.218 \\
\hline $10^{*}$ & 0.201 & 0.920 & 0.032 & 0.013 & 0.851 & 0.053 & 0.005 & 1.111 & 0.054 & 0.039 \\
\hline 10 & 0.036 & 0.916 & 0.080 & 0.291 & & & & 2.548 & 1.077 & 0.151 \\
\hline
\end{tabular}



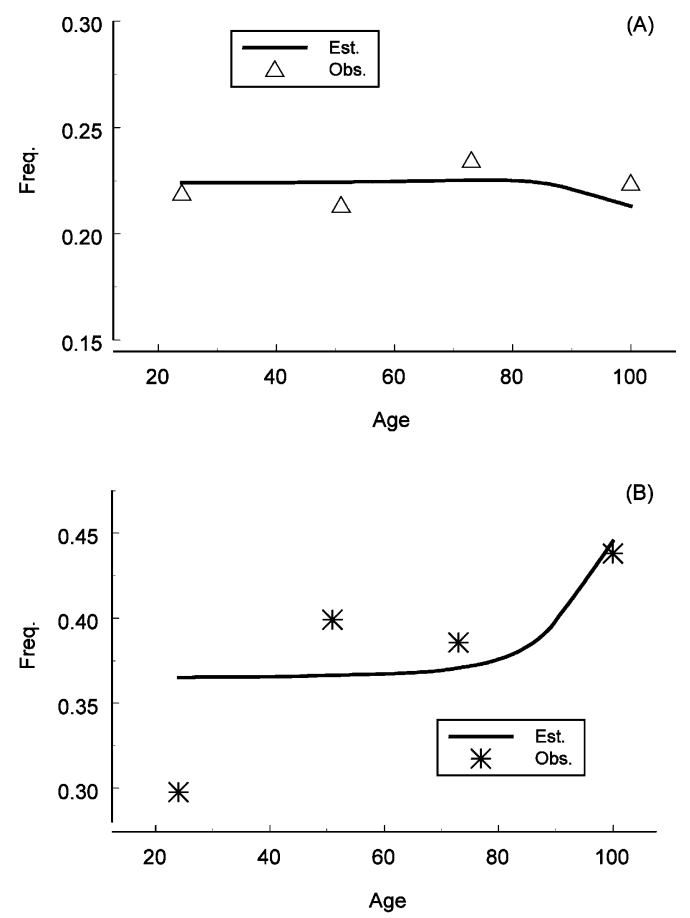

Fig. 1. Observed and estimated frequencies for carriers of allele 8 (A) and allele $10^{*}$ (B) by age.

for $10^{*}$ allele carriers climbs up as age advances (Fig. 1B) revealing obvious survival advantages for $10 *$ allele carriers.

As for the other alleles, allele 9 seems to be recessive and detrimental, because the homozygotes for this allele tend to have increased hazard of death over the ages $(r=1.409$ with $\mathrm{SE}=0.178$, $P=0.022$ ). However, such a risk value becomes non-significant after adjustment for multiple comparisons.

Lastly, as for gene-sex interaction, we see from Table 2 that alleles 6,8 seem to decrease and allele $10^{*}$ to increase the risks of death in males. However, also in this case, the $P$ values become non-significant after adjustment for multiple comparisons.

Note in Table 2, we only estimate one risk for carriers of allele 10 (non-significant) because we only observe one homozygous carrier of the allele in the sample (Table 1).

\subsection{Simulation}

In order to check the performance of our method, a simulation study has been performed by generating 10,000 data sets having exactly the same data structure (size, age and sex compositions) as our sample. To be simple, we assume there is one allele at a locus with relative risk on hazard of death $r=0.9$ for heterozygous carriers and $r^{2}=0.81$ for homozygous carrier. We also assume the allele has no sex-specific effect in that $r_{\mathrm{g} \times \mathrm{s}}=1$. Frequency for the allele is set to 0.2 . For each of the 10,000 data sets, the method described above is applied to retrieve the parameters. Although we assume the risk of heterozygous genotype is the square root of the risk of homozygous genotype in the simulation, we assign two irrelevant risk parameters for the two genotypes in the estimation to see if our model can correctly capture their corresponding true values. Fig. 2 presents the results. The median of allele frequency from the 10,000 estimates is 0.200 and $95 \%$ range $\{0.180,0.220\}$ (Fig. 2A). The medians for estimates on relative risks are 0.895 for heterozygous genotype with $95 \%$ range $\{0.822,0.970\}, 0.801$ for homozygous genotype with $95 \%$ range $\{0.687$, $0.934\}$ and 1.001 for gene-sex interaction with $95 \%$ range $\{0.875,1.138\}$ (Fig. $2 \mathrm{~B}-\mathrm{D}$ ). All the parameter estimates converge to their true values set in the simulation. It is interesting to see that the medians of risk estimates on heterozygous and homozygous carriers of the allele are related as $0.895^{2}=0.801$. This shows again that the model retrieves the parameters used for the simulation.

\section{Discussion}

Given the complexity of the longevity trait, a demanding issue is to develop new models that are capable to capture the various gene trajectories relating to the trait which occur as the population ages and survival selection operates. The present study could serve as a hint in this direction, because it introduces an analytical method which permits to reveal the interaction between the alleles of a gene (TH) associated with human longevity. The method is applied to a large data set, so that 

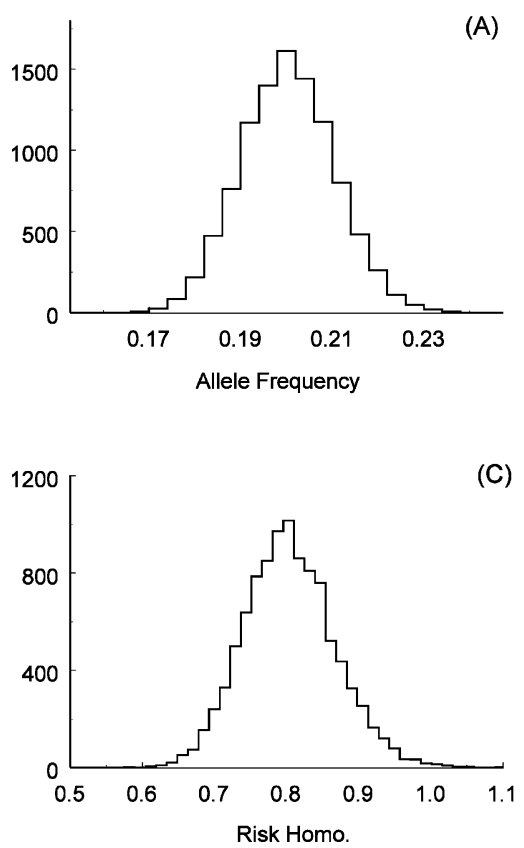

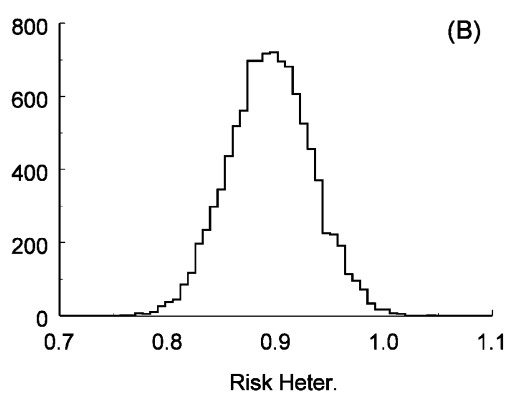

(D)

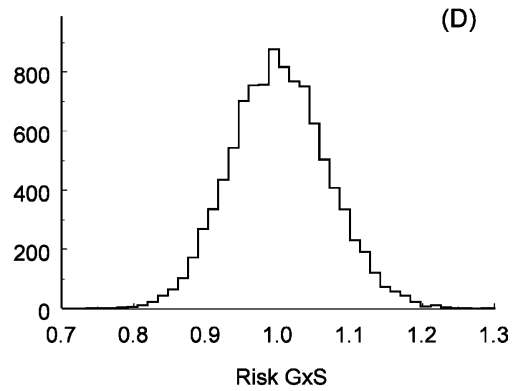

Fig. 2. Parameter estimates from 10,000 simulated data for: (A) allele frequency (true value 0.2); (B) risk of heterozygous genotype (true value 0.9); (C) risk of homozygous genotype (true value 0.81); and (D) risk of gene-sex interaction (true value 1).

subtle effects of the gene variant on the complex trait could be revealed. The basic statistical model described in this paper has been introduced in recent publications (Yashin et al., 1998, 1999b, 2000; Tan et al., 2001b,c). However, the parameterization in the present application shows the feasibility of detecting an allele-allele interaction (see Table 2 and Fig. 1) which implies dominant and additive beneficial effects on the risk of death (allele 10*). An important feature of this application is the combination of individual genetic data with population demographic information in determining the relative risks of the observed genes. The baseline hazard function in this approach is estimated non-parametrically so that no specific survival distribution is imposed on the likelihood function (Tan et al., 2001b). Parameter estimates in this procedure depend heavily on the proportional hazard assumption similar to that in Cox's proportional hazard model. Problems of such an assumption when applied to the genetics of human longevity are discussed in Yashin et al. (1999b), Tan et al. (2001b). However, it can be seen from this study that the proportional hazard assump- tion enables the parameterization for disentangling the various components that are functioning towards longevity, even if the phenotypic effects sustained by the gene on the trait were very subtle (for example, beneficial effects sustained by allele $10 *)$.

The present approach confirms that $\mathrm{TH}$ variability is implicated in human longevity, and in an allele-specific way. How could these effects be explained from a biological point of view? TH encodes the rate-limiting enzyme for the synthesis of cathecolamines, aminoacid-derived molecules that act both as hormones (adrenalin) and neurotransmitters (dopamine and noradrenalin). The $\mathrm{TH}$ gene is therefore a master gene in the neuroendocrine system (NES). Vertebrates have developed a complex hierarchy of multi-organ NES, integrated with the immune system to form the immune-NES. This system governs the capability to cope with a variety of stress-factors and, consequently, rate and quality of aging (Franceschi et al., 2000). The HUMTHO1 microsatellite acts, in vitro, as a transcription regulatory element showing a silencing effect which correlates with the 
number of repetitions (Meloni et al., 1998; Albanese et al., 2001). In particular, the inhibition is proportional to the number of repeats up to allele 8 , while by increasing the number of repeats up to 10 , no further inhibitory effect is observed, but rather a relative enhancement depending on the cell lines used (Albanese et al., 2001). These findings support the idea that HUMTHO1 microsatellite regulates $\mathrm{TH}$ transcription in an allelicspecific way, and modulates the activity of the TH gene acting as a quantitative genomic effector. These allele-specific effects would explain the dominant and dose-dependent beneficial effects we observed for allele $10^{*}$ by our approach. This effect is not found for allele 10, likely because its low frequency with respect to the sample size. On the other hand, a large amount of data suggest the importance of an efficient production of cathecolamines in healthy aging and longevity. Indeed, there are now numerous studies demonstrating that sympathetic factors play a central role not only in cardiovascular homeostatic control, but also in pathogenesis and/or in the progression of several cardiovascular diseases, such as essential hypertension (Rahn et al., 1999) and myocardial infarction (Bertel et al., 1982). Therefore, a good efficiency in TH gene transcription may be a key for an appropriate response towards stochastic and age-related stress factors, and consequently for longevity.

On the whole, the results confirm that the biological background of the aging process is really complex, even if a single locus is considered. With more molecular data becoming available, we hope that the model here presented could help to locate important genes that reside in the genetic realm of human longevity.

\section{Acknowledgements}

This work was supported by the Italian Ministry of University and Technological and Scientific Research (COFIN 2000-2000, Genetic Determinants in Human Longevity), by the Italian Ministry of Health (IRCCS 2000-2002, Marcatori genetici e biologici di invecchiamento normale e patologico), by Istituto Nazionale Riposo Cura Anziani (IN-
RCA), Ancona, Italy, and by grant P01 AG0876101 from the National Institute of Health/National Institute on Aging.

\section{References}

Albanese, V., Faucon Biguet, N., Klefer, H., Bayard, E., Mallet, J., Meloni, R., 2001. Quantitative effects on gene silencing by allelic variation at a tetranucleotide microsatellite. Hum. Mol. Genet. 10, 1785-1792.

Aptech System, 1996. Gauss: Mathematical and Statistical System, vol. I: System and Graphics Manual. Aptech Systems, Maple Valley, WA.

Bertel, O., Buhler, F.R., Baitsch, G., Ritz, R., Burkart, F., 1982. Plasma adrenaline and noradrenaline in patients with acute myocardial infarction. Relationship to ventricular arhythmias of varying severity. Chest 82, 64-68.

De Benedictis, G., Tan, Q., Jeune, B., Christensen, K., Ukraintseva, S.V., Bonafe, M., Franceschi, C., Vaupel, J.W., Yashin, A.I., 2001. Recent advances in human genelongevity association studies. Mech. Ageing Dev. 122, 909920.

De Benedictis, G., Carotenuto, L., Carrieri, G., De Luca, M., Falcone, E., Rose, G., Cavalcanti, S., Corsonello, F., Feraco, E., Baggio, G., Bertolini, S., Mari, D., Mattace, R., Yashin, A.I., Bonafe, M., Franceschi, C., 1998. Gene/ longevity association studies at four autosomal loci (REN, THO, PARP, SOD2). Eur. J. Hum. Genet. 6, 534-541.

De Luca, M., Rose, G., Bonafè, M., Garasto, S., Greco, V., Weir, B.S., Franceschi, C., De Benedictis, G., 2001. Sexspecific longevity associations defined by Tyrosine hydroxylase-insulin-insulin growth factor 2 haplotypes on the 11p15.5 chromosomal region. Exp. Gerontol. 36, 16631671.

Evans, M., Hastings, N., Peacock, J.B., 1993. Statistical Distributions, Second edition. Wiley Interscience, New York, 106-107.

Franceschi, C., Valensin, S., Bonafè, M., Paolisso, G., Yashin, A.I., Monti, D., De Benedictis, G., 2000. The network and remodeling theories of aging: historical background and new perspectives. Exp. Gerontol. 35, 879-896.

Herskind, A.M., McGue, M., Holm, N.V., Sorensen, T.I., Harvald, B., Vaupel, J.W., 1996. The heritability of human longevity: a population-based study of 2872 Danish twin pairs born 1870-1900. Hum. Genet. 97, 319-323.

Kobayashi, K., Kaneda, N., Ichinose, H., Kishi, F., Nakazawa, A., Kurosawa, Y., Fujita, K., Nagatsu, T., 1988. Structure of the human tyrosine hydroxylase gene: alternative splicing from a single gene accounts for generation of four mRNA types. J. Biochem. 103, 907-912.

McGue, M., Vaupel, J.W., Holm, N., Harvald, B., 1993. Longevity is moderately heritable in a sample of Danish twins born 1870-1880. J. Gerontol. 48, B237-B244.

Meloni, R., Albanese, V., Ravassard, P., Treilhou, F., Mallet, J., 1998. A tetranucleotide polymorphic microsatellite, 
located in the first intron of the tyrosine hydroxylase gene, acts as a trascription regulatory element in vitro. Hum. Mol. Genet. 7, 423-428.

Polymeropoulos, M.H., Xiao, H., Rath, D.S., Merril, C.R., 1991. Tetranucleotide repeat polymorphism at the human tyrosine hydroxylase gene (TH). Nucl. Acids Res. 19, 3753.

Puca, A.A., Daly, M.J., Brewster, S.J., Matise, T.C., Barrett, J., Shea-Drinkwater, M., Kang, S., Joyce, E., Nicoli, J., Benson, E., Kunkel, L.M., Perls, T., 2001. A genome-wide scan for linkage to human exceptional longevity identifies a locus on chromosome 4. Proc. Natl. Acad. Sci. USA 98, $10505-10508$.

Rahn, K.H., Barenbrock, M., Hausberg, M., 1999. The sympathetic nervous system in the pathogenesis of hypertension. J. Hypertens. 3, S11-S14.

Tan, Q., Yashin, A.I., De Benedictis, G., Cintolesi, F., Rose, G., Bonafe, M., Franceschi, C., Vach, W., Vaupel, J.W., 2001. A logistic regression model for measuring genelongevity associations. Clin. Genet. 60 (6), 463-469.

Tan, Q., De Benedictis, G., Yashin, I.A., Bonafe, M., DeLuca, M., Valensin, S., Vaupel, J.W., Franceschi, C., 2001. Measuring the genetic influence in modulating human life span: gene-environment and gene-sex interactions. Biogerontology 2, 141-153.

Tan, Q., Yashin, A.I., Bladbjerg, E.M., de Maat, M.P., Andersen-Ranberg, K., Jeune, B., Christensen, K., Vaupel, J.W., 2001. Variations of cardiovascular disease associated genes exhibit sex-dependent influence on human longevity. Exp. Gerontol. 36 (8), 1303-1315.

Tan, Q., Yashin, I.A., Bladbjerg, E.M., Maat, P.M., AndersenRanberg, K., Jeune, B., Christensen, K., Vaupel, J.W., 2002.
A case-only approach for assessing gene by sex interaction in human longevity. J. Gerontol. Biol. Sci., A Biol. Sci. Med. Sci. 57 (4), B129-B133.

Tan, Q., De Benedictis, G., Ukraintseva, S.V., Franceschi, C., Vaupel, J.W., Yashin, A.I., 2002. A centenarian-only approach for assessing gene-gene interaction in human longevity. Eur. J. Hum. Genet. 10 (2), 119-124.

Vaupel, J.W., Yashin, A.I., 1985. Heterogeneity's ruses: some surprising effects of selection on population dynamics. Am. Stat. 39, 176-185.

Yashin, A.I., Vaupel, J.W., Andreev, K.F., Tan, Q., Iachine, I.A., Carotenuto, L., De Benedictis, G., Bonafe, M., Valensin, S., Franceschi, C., 1998. Combining genetic and demographic information in population studies of aging and longevity. J. Epidemiol. Biostatist. 3, 289-294.

Yashin, A.I., Iachine, I.A., Harris, J.R., 1999. Half of the variation in susceptibility to mortality is genetic: findings from Swedish twin survival data. Behav. Genet. 29, $11-$ 19.

Yashin, A.I., De Benedictis, G., Vaupel, J.W., Tan, Q., Andreev, K.F., Iachine, I.A., Bonafe, M., DeLuca, M., Valensin, S., Carotenuto, L., Franceschi, C., 1999. Genes, demography, and life span: the contribution of demographic data in genetic studies on aging and longevity. Am. J. Hum. Genet. 65, 1178-1193.

Yashin, A.I., De Benedictis, G., Vaupel, J.W., Tan, Q., Andreev, K.F., Iachine, I.A., Bonafe, M., DeLuca, M., Valensin, S., Carotenuto, L., Franceschi, C., 2000. Genes and longevity: lessons from studies on centenarians. J. Gerontol. 55a, B319-B328. 